\title{
Observation of nonlinear self-trapping in triangular photonic lattices
}

\author{
Christian R. Rosberg, Dragomir N. Neshev, \\ Andrey A. Sukhorukov, Wieslaw Krolikowski, and Yuri S. Kivshar \\ Nonlinear Physics Centre and Laser Physics Centre, \\ Research School of Physical Sciences and Engineering, \\ Australian National University, Canberra ACT 0200, Australia
}

\begin{abstract}
We experimentally study light self-trapping in triangular photonic lattices induced optically in nonlinear photorefractive crystals. We observe the formation of two-dimensional discrete and gap spatial solitons originating from the first and second bands of the linear transmission spectrum.

(C) 2006 Optical Society of America

OCIS codes: $190.4420,190.5940$
\end{abstract}


Photonic crystals are expected to find many applications in modern optical technologies because of their unique properties arising from the effects of micro-periodicity. In particular, the photonic bandgaps allow one to strongly modify and even suppress the propagation of light in certain directions and at certain frequencies. ${ }^{1}$ Therefore photonic crystals and periodic structures in general offer a wealth of new possibilities to efficiently manipulate the flow of light in optical systems.

Two-dimensional periodic photonic structures can be fabricated in various geometries. Among them triangular lattices with a hexagonal lattice symmetry are known to support larger bandgaps and, therefore, most of the currently fabricated planar photonic structures possess the hexagonal symmetry. Furthermore, the triangular lattice geometry appears naturally in the stacking method fabrication of photonic crystal fibers. ${ }^{2}$

Embedding structural defects into otherwise regular periodic structures allows for increased functionality and the realization of photonic crystal waveguides ${ }^{3}$ and high-Q optical cavities. ${ }^{4}$ In both of these cases, as well as in photonic crystal fibers, light is bound to a lattice defect by modified total internal reflection or Bragg reflection from the surrounding bandgap structure. Efficient trapping, however, requires careful engineering and high accuracy fabrication of the periodic structure as well as the embedded defects, and this typically represents a limiting factor in the practical realization of such devices.

Alternatively, beam self-localization can be used to dynamically introduce refractive index defects in lattice structures with a strong nonlinear response, resulting in the formation of spatial lattice solitons. ${ }^{5}$ Such an approach avoids the need for embedded structural defects to trap the light, and shows inherent advantages for all-optical applications due to the nonlinearity-induced beam control. Efficient nonlinear directional transport ${ }^{6}$ and immobile localizations $^{7}$ were recently demonstrated experimentally for square lattices. Nonlinear mobility and localization of light in triangular lattices, however, remains largely unexploited. In this Letter, we report on the first experimental study of nonlinear light self-localization in triangular lattices in the form of discrete and gap solitons. ${ }^{5}$ We demonstrate that the self-localized beams are immobile and can be considered a nonlinear equivalent of high-Q cavities in photonic crystals.

In experiment, we optically induce a two-dimensional triangular lattice [see Fig. 2(a)] in a biased photorefractive SBN:60 crystal by interfering three ordinarily polarized broad beams from a frequency-doubled $\mathrm{Nd}: \mathrm{YVO}_{4} \mathrm{cw}$ laser. The experimental setup resembles those de- 
scribed earlier. ${ }^{6,8}$ Because of strong electro-optic anisotropy the lattice writing beams propagate linearly in the crystal while extraordinarily polarized probe beams simultaneously experience the induced periodic potential and a strong photorefractive self-focusing nonlinearity. $^{9}$

The propagation of an optical beam along the triangular lattice is governed by the parabolic equation for the slowly varying amplitude of the electric field $\mathrm{d}^{7-9}$

$$
i \frac{\partial E}{\partial z}+D\left(\frac{\partial^{2} E}{\partial x^{2}}+\frac{\partial^{2} E}{\partial y^{2}}\right)+\mathcal{F}\left(x, y,|E|^{2}\right) E=0,
$$

where $x$ and $z$ are the transverse and propagation coordinates normalized to the characteristic values $x_{s}=1 \mu m$ and $z_{s}=1 m m$, respectively, $D=z_{s} \lambda /\left(4 \pi n_{0} x_{s}^{2}\right)$ is the diffraction coefficient, $\lambda$ is the wavelength in vacuum, and $n_{0}$ is the average refractive index of the medium. The function $\mathcal{F}\left(x, y,|E|^{2}\right)=-\gamma\left(I_{b}+I_{p}(x, y)+|E|^{2}\right)^{-1}$ characterizes the total refractive index modulation induced by the optical lattice and the probe beam. Here $I_{b}=1$ is the normalized constant dark irradiance, $I_{p}(x, y)=I_{g} \mid \exp (i k x)+\exp (-i k x / 2-i k y \sqrt{3} / 2)+$ $\left.\exp (-i k x / 2+i k y \sqrt{3} / 2)\right|^{2}$ is the three-wave interference pattern which induces a triangular lattice [see Fig. 1(c)] with period along the $x$ axis $d=4 \pi /(3 k), I_{g}$ is the lattice intensity, and $\gamma$ is a nonlinear coefficient proportional to the applied dc field. ${ }^{10}$ The parameters are chosen to match the experimental conditions: $n_{0}=2.35$ is the refractive index of the bulk photorefractive crystal, $\lambda=532 \mathrm{~nm}$ is the laser wavelength in vacuum, the lattice period is $d=23 \mu \mathrm{m}$ or $d=30 \mu \mathrm{m}, \gamma=2.36$, and $I_{g}=0.49$. The applied electric field is $5 \mathrm{kV} / \mathrm{cm}$.

Light propagation in the linear regime is characterized by the spatially extended eigenmodes or Bloch waves. ${ }^{1}$ The Bloch waves are found as solutions of the linearized equation (1) in the form $E=\psi(x, y) \exp \left(i \beta z+i K_{x} x+i K_{y} y\right)$, where $\psi(x, y)$ possesses the same periodicity as the underlying lattice. The dispersion relations $\beta\left(K_{x}, K_{y}\right)$ are periodic and fully defined by their values in the first irreducible Brillouin zone, ${ }^{11}$ shown in Fig. 1(a). The calculated bandgap spectrum is shown in Fig. 1(b) for lattice period $d=30 \mu \mathrm{m}$. The lattice exhibits a full two-dimensional bandgap for typical experimental parameters.

In a self-focusing medium, the nonlinear response increases the beam propagation constant, shifting it inside the gaps for modes associated with the top of the dispersion bands (i.e. points with maximum $\beta$ ), and allowing for the formation of self-trapped waves or spatial solitons. ${ }^{5}$ It follows that in a triangular lattice, solitons can originate, in particular, from the $\Gamma$ point at the top of the first band (discrete solitons ${ }^{9}$ ) and the $\mathrm{Y}$ point at the top of the 
second band (gap solitons ${ }^{12}$ ) [see Fig. 1(b)].

In order to excite both types of self-trapped waves in the experiment, we shape the probe beams so as to approximate the symmetry of the Bloch waves associated with the corresponding points in the linear transmission spectrum. The calculated Bloch wave at the top of the first band ( $\Gamma$ point) is shown in Fig. 1(d). It exhibits a strong intensity modulation with peaks coinciding with those of the lattice [see Fig. 1(d)], and a constant phase in the transverse plane. This fundamental Bloch wave is excited by a Gaussian beam focused onto a single lattice site at the input face of the crystal [Fig. 2(b)]. The spectral components of the input beam are centered around the $\Gamma$ point in Fourier space [Fig. 1(a) and Fig. 2(c)]. At low laser power $(10 \mathrm{nW})$ the beam experiences discrete diffraction, and most of the light is coupled out of the central lattice site upon propagation, as shown in the three-dimensional plot of the output beam intensity distribution in Fig. 2(d). At high laser power $(1 \mu W)$, on the other hand, the beam localizes at the central lattice site, resembling a discrete lattice soliton [see Fig. 2(e)]. Figure 2(f) shows the calculated nonlinear output beam, and the agreement between theory and experiment is good. This observation represents a clear evidence of beam self-trapping in the total internal reflection gap of the triangular lattice.

The calculated Bloch wave associated with the $\mathrm{Y}$ point at the top of the second band is shown in Figs. 1(e,f). It has a more complex intensity and phase structure and represents a state with a reduced symmetry. The phase structure of the second band wave is staggered in the vertical direction with a $\pi$ phase jump between each zigzag shaped intensity band extending in the horizontal direction. The intensity peaks are positioned off-center with respect to the lattice sites.

The second band wave is excited experimentally by a two-beam interference pattern consisting of horizontal fringes with a staggered phase structure along the vertical direction [Fig. 3(a)]. The period of the interference pattern is matched to that of the corresponding Bloch wave, and the spectral components of the two input beams are centered around the Y point in Fourier space [Fig. 1(a) and Fig. 3(b)]. Despite the rather crude approximation to the actual Bloch wave profile, the second band mode is successfully excited in the experiment, as seen in Fig. 3(c) which shows the central part of the linear output from the crystal.

At low power the second band wave strongly diffracts in the lattice [Fig. 3(d)] while at high power it localizes to almost a single lattice site with two out-of-phase and off-center lobes, thus preserving the Bloch wave symmetry. This is verified by interferometric measurements 
revealing a clear $\pi$ phase jump at the center of the localized beam [Fig. 3(f)]. Numerical simulations confirm the observed intensity [Fig. 3(g)] and phase [Fig. 3(h)] structure of the localized beam. Again we find that observations agree well with theory, although some asymmetry and noise in the excited wave is seen in experiment. The observations in Fig. 3 represent a clear evidence of beam self-trapping inside the first Bragg reflection bandgap of the triangular lattice, and as such demonstrates a nonlinear analog to photonic crystal high-Q cavities.

The localized gap state is found to be experimentally robust and immobile in any direction, an observation standing in stark contrast to the strong directional mobility of reducedsymmetry gap solitons in square lattices. ${ }^{6}$

We note that gap solitons can also be associated with the $\mathrm{J}$ point of the second spectral band $^{12}$ [Figs. $\left.1(\mathrm{a}, \mathrm{b})\right]$ where the phase structure at the soliton core is rotated by 30 degrees compared to the Y state. According to numerical calculations, the internal energies of the $\mathrm{Y}$ and J gap solitons are almost identical under our experimental conditions, and transformation between these states could therefore be induced by a small input beam asymmetry. Indeed, the observed second band wave is seen to be slightly rotated at the crystal output.

In conclusion, we have demonstrated experimentally light self-localization and the formation of two-dimensional discrete and gap spatial solitons in optically induced triangular photonic lattices. We believe our results may be useful for other periodic photonic structures with similar geometry such as planar photonic crystals and micro-structured optical fibres.

We acknowledge support from the Australian Research Council. 


\section{References}

1. P. St. J. Russell, T. A. Birks, and F. D. Lloyd Lucas, "Photonic Bloch waves and photonic band gaps," in Confined Electrons and Photons, E. Burstein and C. Weisbuch, eds. (Plenum Press, New York, 1995), pp. 585-633.

2. A. Bjarklev, J. Broeng, and A.S. Bjarklev, Photonic Crystal Fibres (Kluwer/Springer, Dordrecht, 2003).

3. H. Gersen, T.J. Karle, R.J.P. Engelen, W. Bogaerts, J.P. Korterik, N.F. Hulst, van, T.F. Krauss, and L. Kuipers, "Real-Space Observation of Ultraslow Light in Photonic Crystal Waveguides," Phys. Rev. Lett. 94, 073903-4 (2005).

4. B.S. Song, S. Noda, T. Asano, and Y. Akahane, "Ultra-high-Q photonic doubleheterostructure nanocavity," Nature Materials 4, 207-210 (2005).

5. Yu.S. Kivshar and G.P. Agrawal, Optical Solitons: From Fibers to Phootnic Crystals (Academic Press, San Diego, 2003), 540 pp.

6. R. Fischer, D. Trager, D.N. Neshev, A.A. Sukhorukov, W. Krolikowski, C. Denz, and Yu.S. Kivshar, "Reduced-Symmetry Two-Dimensional Solitons in Photonic Lattices," Phys. Rev. Lett. 96, 023905-4 (2006).

7. J.W. Fleischer, M. Segev, N.K. Efremidis, and D.N. Christodoulides, "Observation of two-dimensional discrete solitons in optically induced nonlinear photonic lattices," Nature 422, 147-150 (2003).

8. D. Neshev, E. Ostrovskaya, Y. Kivshar, and W. Krolikowski, "Spatial solitons in optically induced gratings," Opt. Lett. 28, 710-712 (2003).

9. N.K. Efremidis, S. Sears, D.N. Christodoulides, J.W. Fleischer, and M. Segev, "Discrete solitons in photorefractive optically induced photonic lattices," Phys. Rev. E 66, 046602-5 (2002).

10. E. DelRe, B. Crosignani, and P. Di Porto, "Photorefractive spatial solitons," in Spatial Solitons, Vol. 82 of Springer Series in Optical Sciences, S. Trillo and W. E. Torruellas, eds. (Springer-Verlag, New York, 2001), pp. 61-85.

11. G. Bartal, O. Cohen, H. Buljan, J.W. Fleischer, O. Manela, and M. Segev, "Brillouin Zone Spectroscopy of Nonlinear Photonic Lattices," Phys. Rev. Lett. 94, 163902-4 (2005).

12. N. Akozbek and S. John, "Optical solitary waves in two- and three-dimensional nonlinear photonic band-gap structures," Phys. Rev. E 57, 2287-2319 (1998). 


\section{FIGURE CAPTIONS}

Fig. 1. (Color online) (a) Lattice unit cell in Fourier space; (b) Bloch-wave dispersion along the contour passing through the high-symmetry points marked in (a); (c) Refractive-index profile of the triangular lattice; (d,e) Bloch waves corresponding to the $\Gamma$ and $Y$ points in $(\mathrm{a}, \mathrm{b}):(\mathrm{d})$ intensity at the $\Gamma$ point of the $1^{\text {st }}$ band, $(\mathrm{e}, \mathrm{f})$ intensity and phase at the $Y$ point of the $2^{\text {nd }}$ band.

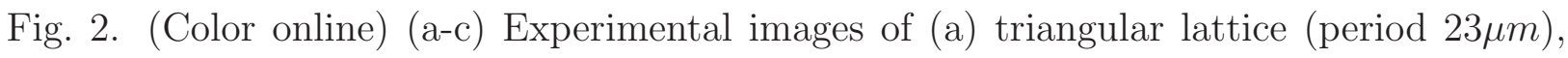
(b) single-beam input intensity distribution, and (c) Fourier spectrum of input and lattice beams. In $(a, b)$ the dashed hexagon indicates the lattice unit cell, and in (c) the edge of the $1^{\text {st }}$ Brillouin zone as defined by the three lattice beams. (d,e) Measured linear discrete diffraction and nonlinear self-trapping, respectively, from the top of the $1^{\text {st }}$ band. The plot dimensions are $150 \mu m$ along both $x$ and $y$. (f) Numerically calculated intensity of discrete soliton.

Fig. 3. (Color online) Experimental images of (a) two-beam input intensity distribution, (b) Fourier spectrum of input and lattice beams, and (c) linear $2^{\text {nd }}$ band Bloch wave at the crystal output. In (b) the dashed hexagon indicates the edge of the $1^{\text {st }}$ Brillouin zone, and in (c) the lattice unit cell. (d,e) Measured linear discrete diffraction [as in (c)] and nonlinear self-trapping, respectively, from the top of the $2^{\text {nd }}$ band. The plot dimensions are $150 \mu m$ along $x$ and $200 \mu m$ along $y$. (f) Measured phase interferogram for the self-trapped beam in (e). $(\mathrm{g}, \mathrm{h})$ Numerically calculated gap soliton intensity distribution and phase, respectively. The lattice period is $30 \mu \mathrm{m}$. 

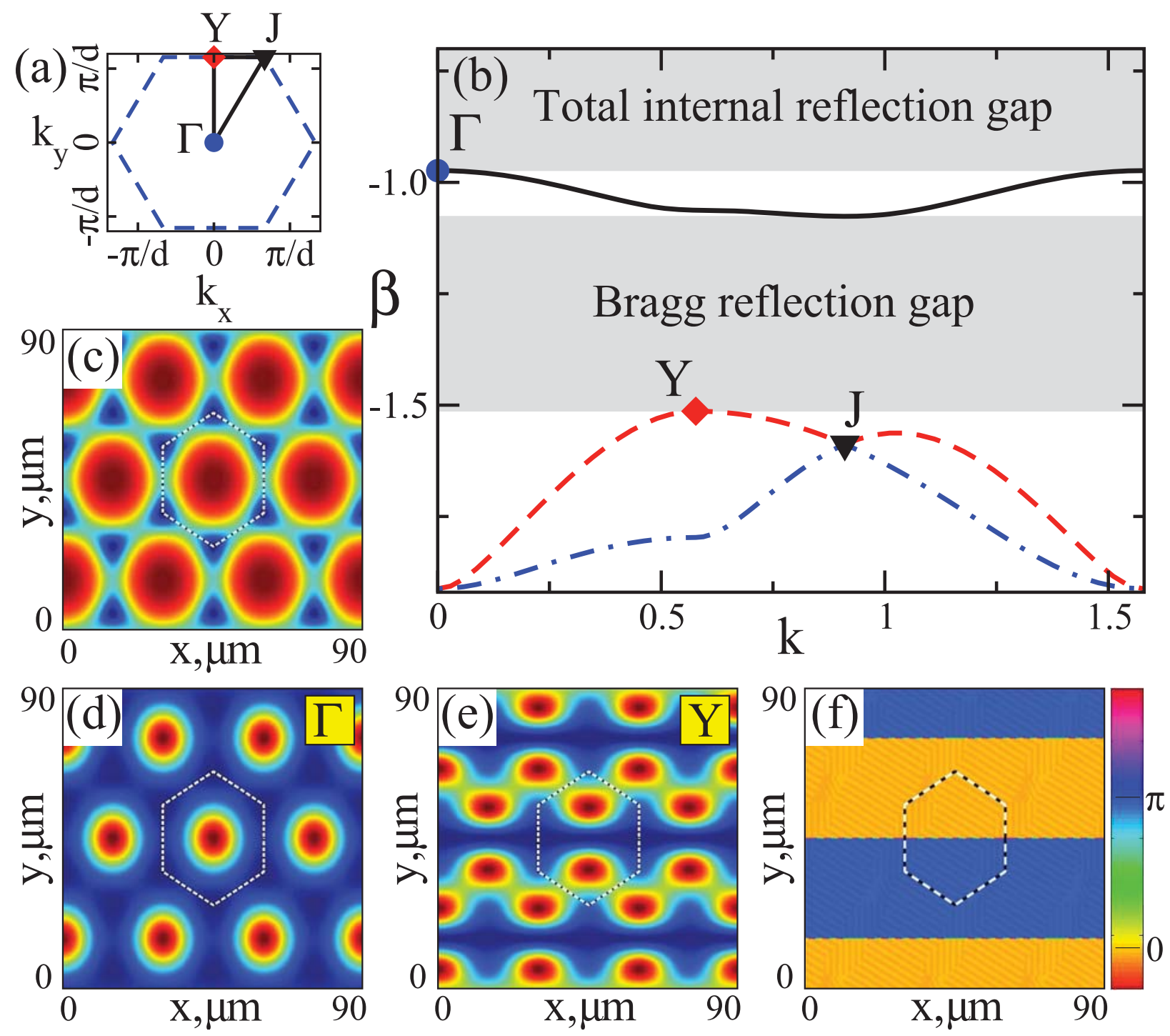

Fig. 1. 

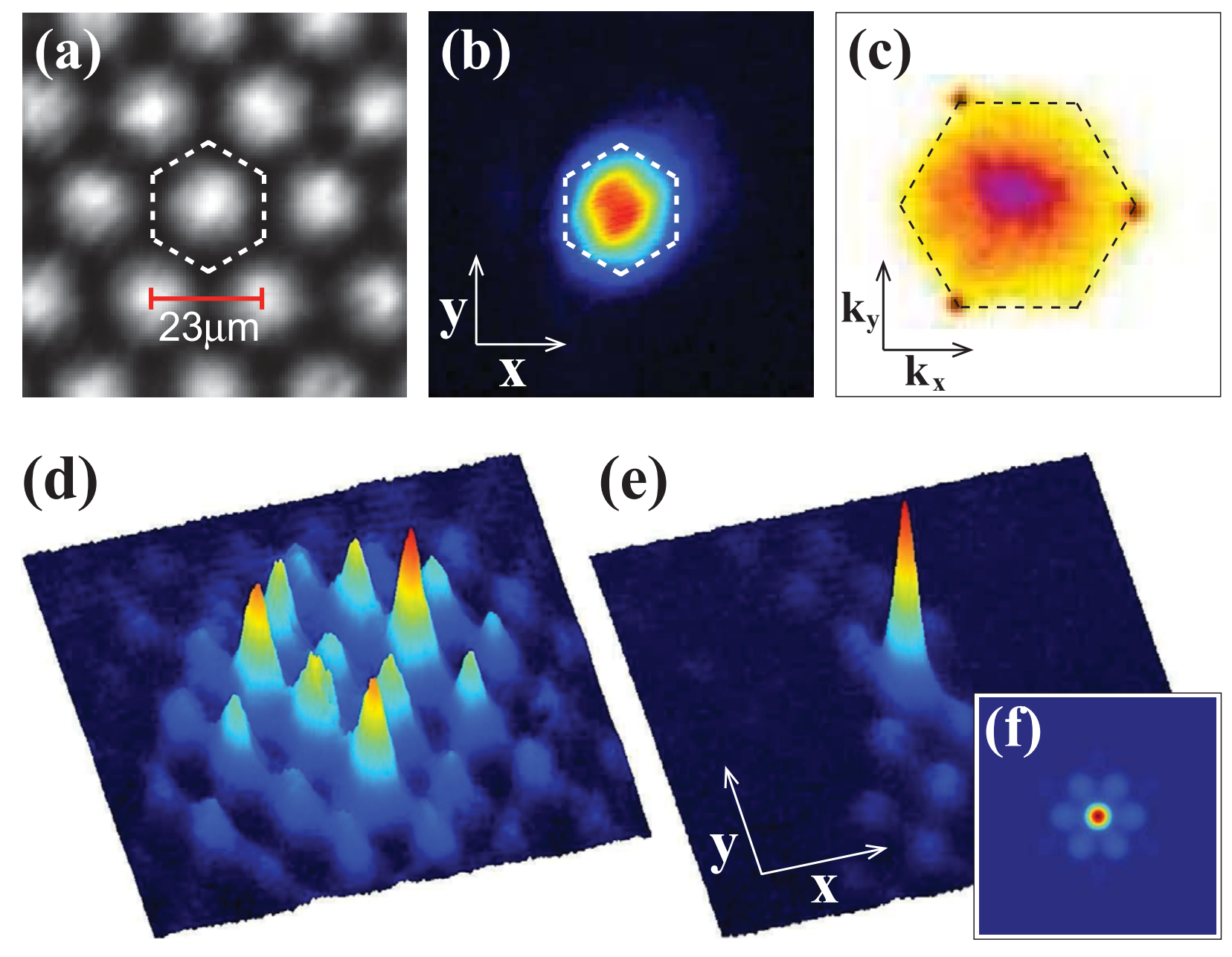

Fig. 2. 

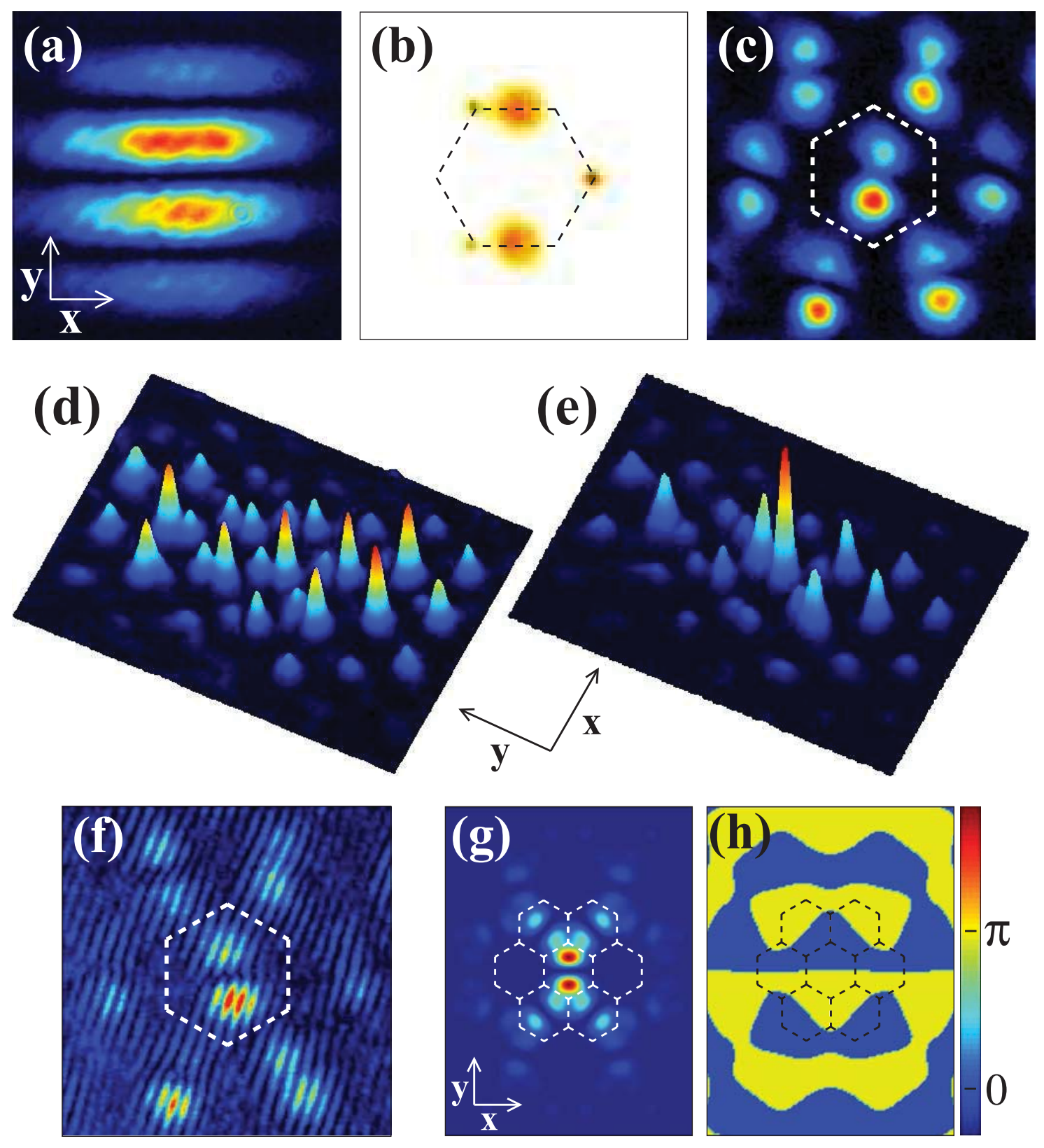

Fig. 3. 